\title{
Characteristics of Femtosecond Laser Pulses Propagating in Multiply Ionized Rare Gases
}

$$
\text { Valer Toșa }{ }^{1,2,5}, \text { Katalin Kovács }^{1 *} \text {, Daniel Ursescu }{ }^{3} \text {, and Katalin Varjú }{ }^{2,4}
$$

${ }^{1}$ National Institute for R\&D of Isotopic and Molecular Technologies, 400293 Cluj-Napoca, Romania

${ }^{2}$ ELI ALPS, ELI-HU Nkft, 6720, Szeged, Hungary

${ }^{3}$ ELI-NP, Horia Hulubei National Institute for R\&D in Physics and Nuclear Engineering, 077125 Magurele,

$$
\text { Romania }
$$

${ }^{4}$ Dept. Optics \& Quantum Electronics, University of Szeged, 6720 Szeged, Hungary

${ }^{5}$ King Saud University, Riyadh-11451, Saudi Arabia

\begin{abstract}
A three-dimensional non-adiabatic model in combination with a rate equation model is used to characterize high-intensity $\left(>10^{16} \mathrm{~W} / \mathrm{cm}^{2}\right)$ femtosecond laser pulse propagation in atomic gas medium. In these conditions the atoms in the target gas will become multiply charged ions, while the laser pulse will propagate in a medium with high electron concentration created by itself. We obtain pulse characteristics in time, frequency and space domain for two representative cases: $800 \mathrm{~nm}$ and $267 \mathrm{~nm}$ (third harmonic) multicycle pulses which are both of practical importance. We show that the spatial-temporal variation of the refractive index in the macroscopic medium is the primary reason for pulse temporal/spectral and spatial shaping during propagation.
\end{abstract}

Keywords: high-intensity laser pulse; propagation equation; highly ionized atomic medium; propagation effects;

*corresponding author: kkovacs@itim-cj.ro

National Institute for R\&D of Isotopic and Molecular Technologies, 67-103 Donat str. 400293 Cluj-Napoca, Romania 


\section{Introduction}

Very intense laser pulses, approaching the relativistic intensity regime are already, or will be soon available in many laser laboratories and infrastructures. Studying the propagation properties of intense laser pulses in ionizing media is important in a wide range of applications, including laser-driven particle acceleration [1], laser-plasma channeling [2], high order harmonic generation (HHG) [3], supercontinuum generation [4], Xray lasers and laser-fusion schemes (see review [1]).

When an ultrashort, intense laser pulse is used in particle acceleration experiments, spatial and energy distributions of energetic electrons are shown $[5,6]$ to depend strongly on the contrast ratio and shape of the laser prepulse. Moreover, in electron acceleration experiments it was found that a stable, long distance propagation of ultrahigh intensity laser pulses through a He plasma is a critical issue to produce stable multi$\mathrm{GeV}$ electron beams [6]. For this reason the distortions induced in the temporal and spatial domain of an ultraintense pulse should be quantified both for characterizing the pulse and for estimating the electron density map and its time evolution [6]. Solving the pulse propagation problem at intensities found in the temporal and spatial pedestals of ultraintense pulses can yield valuable information in understanding particle acceleration experiments. Recently, PIC simulation results concerning laser-plasma interaction in the subrelativistic regime have been reported [7] and pointed out how important the pulse shaping (temporal and spectral) during propagation is for a correct description of the complex mechanisms taking place in the interaction region.

High-order harmonic generation (HHG) is an intensively investigated method for converting infrared or mid-infrared laser pulses towards XUV or soft-X-ray ultrashort coherent pulses with high photon flux. The presence of electrons in the gas medium where HHG takes place is a reality which cannot be overruled, as in these experiments one produces orders of magnitude more electrons than photons. The rapidly increasing laser pulse powers give promise for HHG in increasing magnitude, if macroscopic conditions can be chosen suitably. One option for scaling up HHG is a geometrical enlargement [8], keeping the system in the low ionization regime. However, the peak powers to be available in the new laser facilities - like ELIBeamlines (Prague, Czech Republic) 10 PW, ELI-ALPS (Szeged, Hungary) 1-3 PW, ELI-NP 
(Magurele, Romania) 2x10 PW, IBS-CoReLS (Gwangju, Korea) 4 PW - will be so high that alternative methods of utilizing highly ionized arrangements are proposed.

A recent direction of research is to exploit the formation of multiply ionized gases by using as driving field the compressed pulse of a Ti:Sa laser [9], or its third harmonic [10]. In this case the dynamics of plasma in time and space is one of increasing complexity and the harmonic emission can be obtained from different ionic species. As outlined in [10], a macroscopic modeling of HHG in multiply ionized media is imperative in order to understand the whole process in these special conditions.

Laser pulses of $>10^{16} \mathrm{~W} / \mathrm{cm}^{2}$ intensity generated in Ti:Sa at $800 \mathrm{~nm}$ central frequency are nowadays obtainable in many laboratories. In this paper we present two cases of practical importance. (1) The $800 \mathrm{~nm}$ ultraintense pulse propagates in noble gas atomic medium with the ultimate goal to generate high-order harmonics through the laser-atom interaction, the resulting radiation being highly coherent in the XUV and even in the soft X-ray region. At this intensity the atoms in the generating medium will multiply ionize, which was not yet studied in current models. (2) The second possibility is to let the third harmonic of the $800 \mathrm{~nm}(267 \mathrm{~nm})$ interact with the atomic medium. Third harmonic generation is a widely used parametric process and has $\sim 10 \%$ efficiency. At the moment we restrict our study to the propagation of the two laser pulses $(800 \mathrm{~nm}$ and $267 \mathrm{~nm})$ in Ar gas in a single-shot mode: we track the ionization dynamics of the gas both in temporal and spatial resolution, and show the spatial, temporal and spectral modifications of the generating laser pulse itself.

\section{Model}

Propagation of an ultrashort pulse in an ionizing gas medium can be described in cylindrical coordinates by the equation [11]:

$\nabla^{2} E_{1}(r, z, t)-\frac{1}{c^{2}} \frac{\partial^{2} E_{1}(r, z, t)}{\partial t^{2}}=\frac{\omega^{2}}{c^{2}}\left(1-\eta_{e f f}^{2}\right) E_{1}(r, z, t)$,

where $c$ is the speed of light, $E_{1}(r, z, t)$ is the full electric field (carrier) with central frequency $\omega$, and the effective refractive index is written as 
$\eta_{e f f}\left(n_{a}, n_{e}, r, z, t\right)=\eta_{0}\left(n_{a}\right)+\eta_{2}(t) I(r, z, t)-\frac{\omega_{p}^{2}\left(n_{e}, r, z, t\right)}{2 \omega^{2}}$.

This formula accounts for the dispersion by neutrals of density $\boldsymbol{n}_{\boldsymbol{a}}$ and electron plasma of density $\boldsymbol{n}_{\boldsymbol{e}}$ as well as for the nonlinear refractive index $\eta_{2}$ associated with the bound electrons (optical Kerr effect). The last term of Eq. 2 contains the square of the plasma frequency $\omega_{p}^{2}$ which is proportional to the electron concentration $n_{e}$. In a very intense laser field an atom/molecule experiences multiple ionization. We calculate the electron concentration considering sequential ionization described by the system of rate equations (similar to that used in [12] and [13]) for the calculation of fractions of ionic species:

$\frac{d n_{0}(t)}{d t}=-n_{0}(t) w_{01}(t)$

$\frac{d n_{1}(t)}{d t}=n_{0}(t) w_{01}(t)-n_{1}(t) w_{12}(t)$

$\cdots \cdots$

$\frac{d n_{Z}^{(t)}}{d t}=n_{Z-1}(t) w(Z-1) Z^{(t)}$

Here $\boldsymbol{n}_{\boldsymbol{0}}$ is the initial density of the medium and $w_{(k-1) k}(t)$ are the ionization rates from the $\mathrm{Z}=k-1$ to $\mathrm{Z}=k$ ion; the rates were calculated using the Ammosov-Delone-Krainov (ADK) [14] approximation, while the time-dependent density of the ionic species with charge $\boldsymbol{k}$ is $\boldsymbol{n}_{\boldsymbol{k}}(\boldsymbol{t})$. We solve the system of rate equations starting from the neutral atom up to the completely ionized one. In order to correctly evaluate the variation of the effective refractive index due to the plasma contribution, we calculate the total number of electrons resulting from this consecutive stripping of an atom/molecule as:

$n_{e}(t)=\sum_{k=1}^{Z} k n_{k}(t)$

Based on Eq. (4) we define the time-dependent ionization degree as $n_{e}(t) / n_{0}$. This quantity has nontrivial variation and can take the highest value $Z$ in the case when all atoms (atomic number $Z$ ) in the interaction region are completely ionized.

The optical Kerr term in Eq. 2. requires some more attention in the conditions discussed in this paper. As multiply ionized species are present one has to account for their contribution to the third order susceptibility $\chi_{3}$ of the medium. The values of the susceptibilities for ions are not known but can be estimated. Here we used the relationship that can be established between the susceptibility of the ion $\chi_{3}^{+}$and 
the susceptibility of the neutral atom $\chi_{3}^{0}$, their ratio being in connection with the ratio of their ionization potentials as $\chi_{3}^{+} / \chi_{3}^{0} \approx\left(I_{p}^{0} / I_{p}^{+}\right)^{3}$, where $I_{p}$ stands for the ionization potentials of the species involved [15]. The nonlinear refractive index then takes the form: $\eta_{2}(t)=\sum_{k} \eta_{2}\left(t, n_{k}\right)$.

The imaginary part of the refractive index was also considered in order to estimate the damping of the wave during propagation. We assume that the energy lost by the pulse is solely due to having to supply energy for ionization. In order to find this contribution we extended the method valid for single ionization described in [13] such to account for multiple ionization. In short, we write the energy balance for the ionization process and assume that the energy lost by the pulse between $z$ and $z+d z$ is the opposite of the energy gained by the electrons in the same interval. The energy loss was estimated for each $(r, z)$ point after every successful integration step, and the damped field was used in the next integration step.

We only consider here the propagation of single-shot laser pulses traveling through the Ar medium. It takes 3.3 ps for such a "light bullet" to fly through $1 \mathrm{~mm}$ gas, while the typical plasma decay time is $\sim 100 \mu \mathrm{s}$. In the modeling we let the laser pulses enter an initially neutral medium, and do not take into account the effects of a preceding pulse, which at high repetition rate could influence the distribution of the ionic species in the medium. However, when the generated pulses reach the PW regime, the repetition rate is $\sim 1 \mathrm{~Hz}$ or lower, so the single-shot simulation in the present case study is justified.

In this model the input parameters are closely related to the experimentally measurable quantities, such as laser pulse energy, duration, geometrical focusing conditions, gas type and pressure of the medium. Our calculations yield detailed results about the modifications of the driving laser pulse in spatial dimension, while the temporal resolution is at optical cycle level. We also obtain valuable information about the dynamics of the production and distribution of electrons and ions (of different charge) both in space, covering the whole interaction volume, and in time, spanning the duration of the driving laser pulse.

\section{Results and discussion}

The parameters for the cases studied here are the following:

Case 1: The laser pulse with $\lambda=800 \mathrm{~nm}, \tau=35 \mathrm{fs}$ (intensity full width at half maximum), $\mathrm{E}_{\text {pulse }}=26 \mathrm{~mJ}$ was focused with an $f=0.75 \mathrm{~m}$ optical element into a $2 \mathrm{~mm}$ long cell filled with Ar at 30 Torr pressure, the 
cell entrance situated $1 \mathrm{~mm}$ before the geometrical focus, so that the cell center is in the geometrical focus. This configuration yields a peak intensity at the cell entrance of $1.2 \cdot 10^{16} \mathrm{~W} / \mathrm{cm}^{2}$ and the beam is converging as it reaches the medium with beam diameter of $120 \mu \mathrm{m}$.

Case 2: We use the $3^{\text {rd }}$ harmonic of the previous beam, i.e. $\lambda=267 \mathrm{~nm}, \tau=30 \mathrm{fs}, \mathrm{E}_{\text {pulse }}=2.6 \mathrm{~mJ}$. The rest of the "experimental" configuration was kept unchanged. So, the initial intensity was the same $1.2 \cdot 10^{16} \mathrm{~W} / \mathrm{cm}^{2}$ as for the fundamental beam, and the beam diameter at cell entrance was of $40 \mu \mathrm{m}$. We have chosen this configuration for two reasons: to have experimentally feasible pulses and to be able to start from equal intensities so that the results become directly comparable. We mention that the relativistic parameter $a_{0}$ which is the normalized vector potential of the field is $<10^{-2}$ for this case, meaning that the model presented in the previous section is valid.

In Fig. 1. we show the time evolution of the populations of different ionic species at the cell entrance, i.e. in case of the non-distorted $800 \mathbf{~ n m ~} 35$ fs laser pulse. In order to be able to follow the increasing pulse intensity, we also represent the laser pulse envelope. We start from all neutral Ar atoms in the medium. As the intensity increases the neutral population will be depleted while $\mathrm{Ar}^{+}$ions are created by field ionization. Successively we arrive to completely ionize the medium to $\mathrm{Ar}^{6+}$ around the pulse peak. Given the equivalent initial shape of the two pulses, we obtain the same ion population evolution also for the $3^{\text {rd }}$ harmonic pulse $(267 \mathrm{~nm} 30 \mathrm{fs})$. We must mention that population dynamics obtained for this last case was in good agreement with a similar result obtained in [10] by solving the time dependent Schrödinger equation.

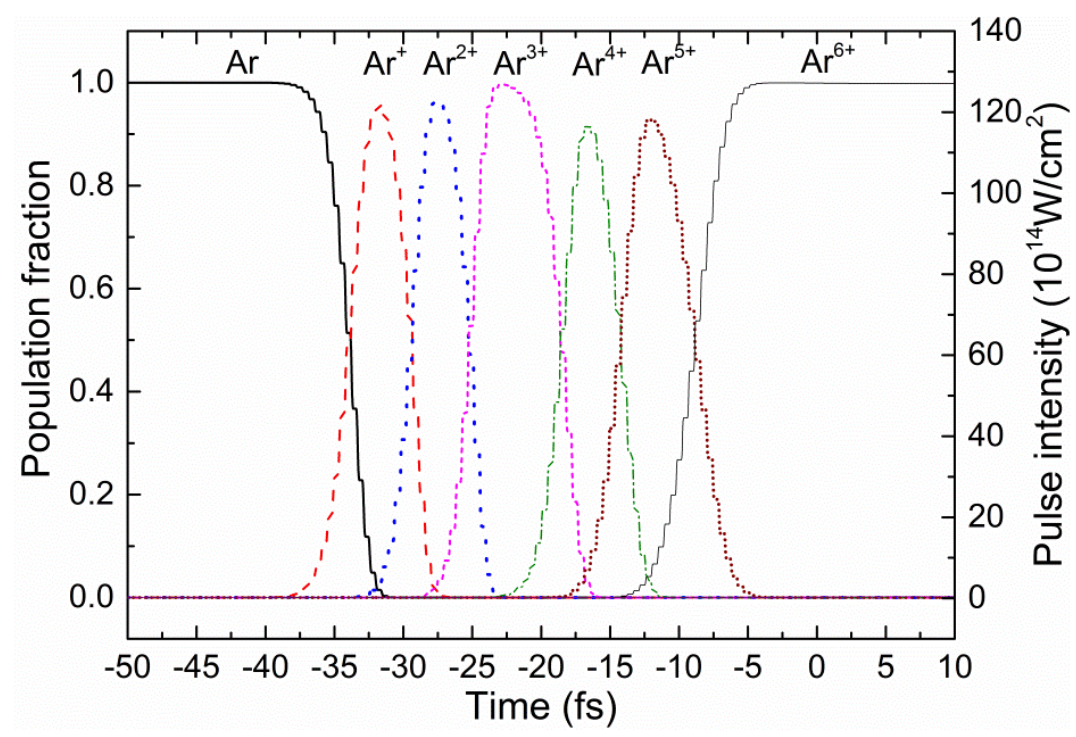

Fig. 1. (color online) 
In Fig. 2. we show the ionization degree (defined as $\boldsymbol{n}_{\boldsymbol{e}}(\boldsymbol{t}) / \boldsymbol{n}_{\boldsymbol{0}}$ ) at the beginning of the propagation (black / top) and after $2 \mathrm{~mm}$ propagation (red / bottom). We also show the full electric fields for both pulses (left 800 $\mathrm{nm}$, right $267 \mathrm{~nm}$ ) at the beginning of propagation and at the exit from the medium. Both pulses (especially the leading edges) are seriously deformed in the plasma medium, the principal source being the rapid variation of the plasma dispersion term in the effective refractive index (last term in Eq. 2.) experienced by each pulse. Regarding the ionization dynamics we see that small ionization steps occur once every half optical cycle at the maximum amplitude of the field, when electrons are extracted from the atom / ion with highest probability. The appearance of ions with $\mathrm{Z}+1$ charge comes after a plateau at $\mathrm{Z}$ charge. The temporal length of such a plateau is determined by the difference between the successive ionization potentials $I_{p(Z+1)^{-}}$ $I_{p(Z)}$.
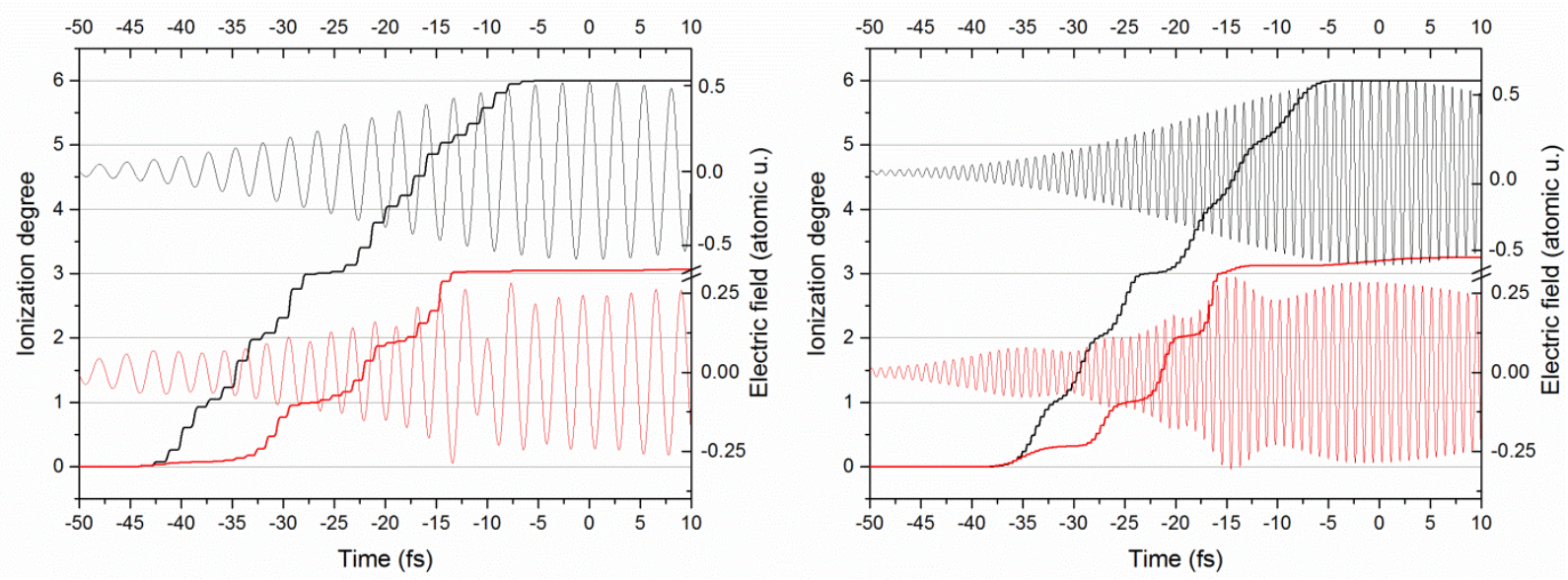

Fig. 2. (color online)

At the beginning of the propagation the initial laser pulses have Gaussian envelopes both in the temporal shape and in the radial distribution. The spatial evolution of the peak intensities during propagation is shown in Fig. 3 for the $800 \mathrm{~nm}$ on the left and for the $3^{\text {rd }}$ harmonic on the right. We observe one high peak in the intensity map in case of the $800 \mathrm{~nm}$ pulse which arises along propagation on the optical axis. In order to clarify the origin of this phenomenon we show in the inset of Fig. 3 the laser pulse envelope in three characteristic positions along propagation indicated with dots: at the entrance of the medium (black), at the position with highest intensity (red) and at cell exit (green). We clearly observe a pulse shortening and a retardation of the pulse due to the high level of ionization. Similar effect has already been reported in experiments performed with laser pulse intensities up to $10^{17} \mathrm{~W} / \mathrm{cm}^{2}$ [16]. Slight intensity increase is 
observable also during the propagation of the $3^{\text {rd }}$ harmonic pulse, but it is much less pronounced because in this case plasma effects and pulse shortening are weaker. We thus came to the conclusion that the pulse steepening is a consequence of the change of the refractive index due to a fast variation in time and a non-

\section{homogeneous variation in space of the plasma density.}

The two pulses have equal waists at the focusing element, therefore the waists in the focus are related as $\mathrm{W}_{0(800)}=3^{*} \mathrm{~W}_{0(267)}$. It is visible on the spatial intensity maps that the radial dimension of the whole propagation scales correspondingly. Moreover, this scaling is preserved also for the spatial distribution of the ionization degree, as represented in Fig. 4.
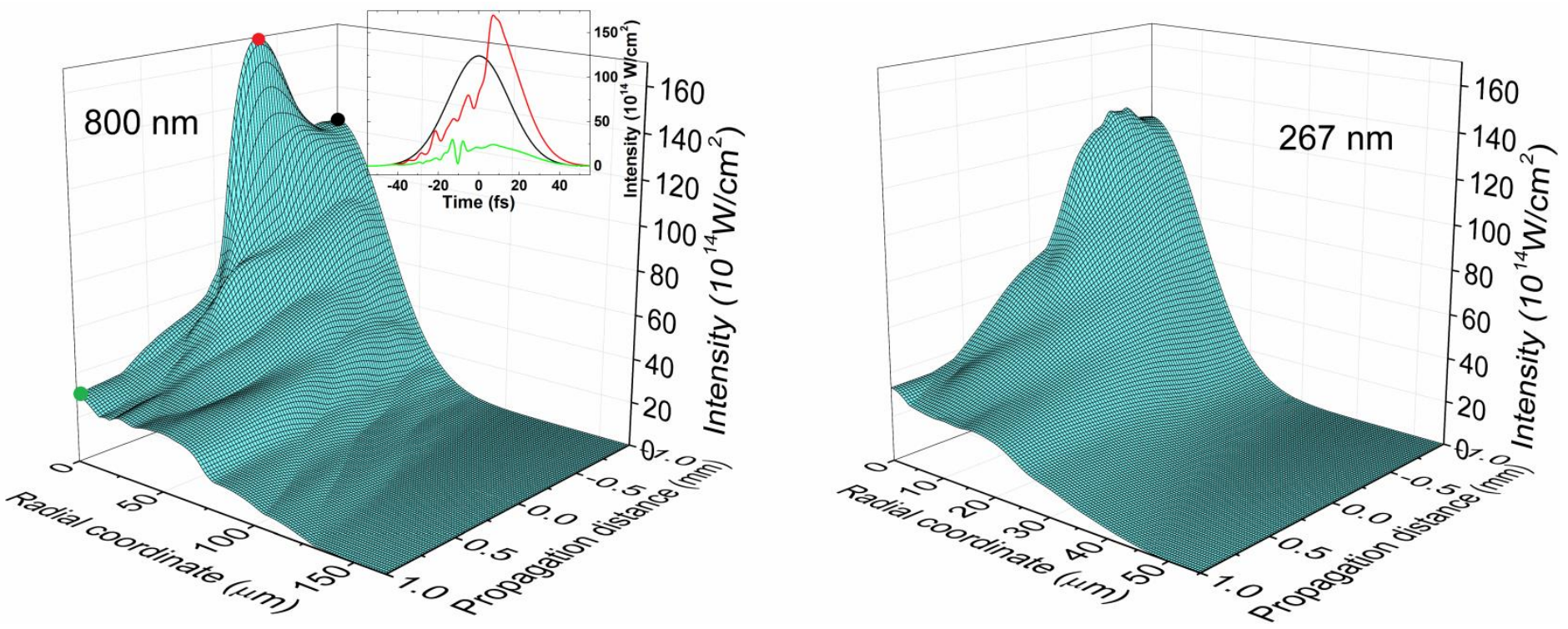

Fig. 3. (color online)
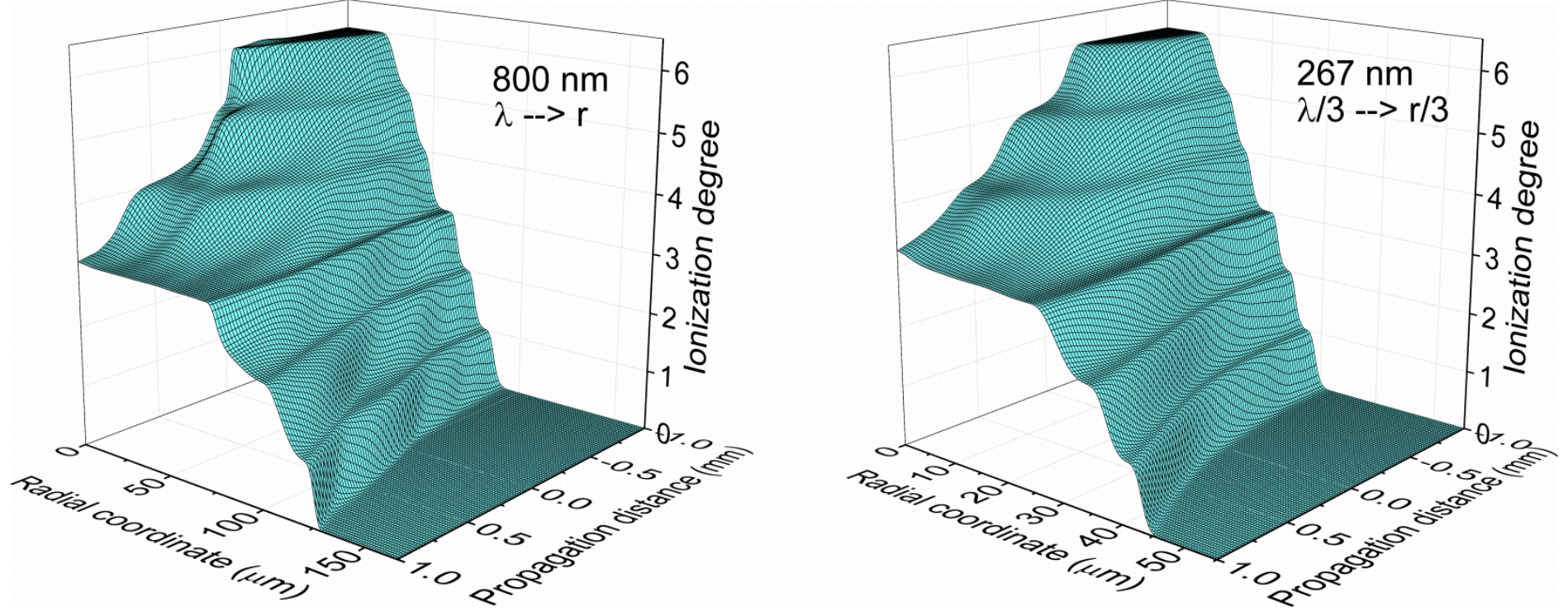

Fig. 4. (color online) 
Fig. 4. shows the spatial map of the ionization degree at pulse end, as it evolves during the pulse propagation in the Ar medium. Each point in the spatial map in Fig. 4. represents the final value of the ionization degree as shown in Fig. 2. In order to have a correct understanding regarding the ionic composition of the medium along propagation we have to interpret Fig. 1 and Fig. 4 together. We observe for example that $\mathrm{Ar}^{3+}$ has a robust development in the $2-4 \cdot 10^{15} \mathrm{~W} / \mathrm{cm}^{2}$ intensity regime. However, in the spatial domain (see Fig. 4) where the 3+ ionization degree forms a "terrace", the ionic content of the medium is a mixture consisting of $\mathrm{Ar}^{2+}, \mathrm{Ar}^{3+}, \mathrm{Ar}^{4+}$. This fact can be correlated with the data in Fig. 1 where it is obvious how the tails of successive ionic distributions overlap. The same situation is expected to persist in the medium after the laser pulse has gone: in the central region in a cylinder of $\sim 60 \mu \mathrm{m}$ radius $(800 \mathrm{~nm}$ case) or $\sim 20 \mu \mathrm{m}$ radius (267 $\mathrm{nm}$ case) the dominant ions are $\mathrm{Ar}^{6+}$, but at the periphery the net charge decreases according to the intensity decrease of the laser pulse which was responsible for the plasma creation.

One very important remark should be made about the ionization degree spatial maps presented in Fig. 4: we observe the striking similarity of the two spatial maps, the only difference being their radial extent, which respects the $\mathrm{w}_{0(\lambda)} \rightarrow 3^{*} \mathrm{w}_{0(\lambda / 3)}$ or the $\lambda \rightarrow \lambda / 3$ scaling taking into account that their waists at the focusing element were set equal. This fact seems to be an extension of the scaling principle of the non-linear effects during laser pulse propagation, as was mathematically shown in [8]. The validity of the scaling is even more robust if we take into account that the fast temporal variation of the effective refractive index in the two cases is different. The Kerr contribution is similar since the starting intensities were set equal. However, the plasma term's temporal evolution differs in the two cases: (1) the $800 \mathrm{~nm}$ pulse is somewhat longer (35 fs vs $30 \mathrm{fs}$ for the $267 \mathrm{~nm}$ ), therefore ionization starts earlier in the leading edge of the pulse; (2) the plasma term in the total refractive index is $\sim \omega^{-2}$, therefore the plasma contribution to the refractive index experienced by the $800 \mathrm{~nm}$ pulse becomes 9 times stronger than the one seen by the $3^{\text {rd }}$ harmonic pulse.

When looking at the spectral modifications of the two laser fields (Fig. 5.) we clearly see that the $800 \mathrm{~nm}$ pulse is more affected by the modifications of the medium's refractive index. The central frequency experiences a blue-shift ( $7 \mathrm{~nm}$ shift), and in addition the whole spectrum of the pulse is broadened toward shorter wavelengths. Ionization-induced blue-shifting of the spectrum has been studied both theoretically 
[13] and experimentally [17]. The spectral blue-shifts obtained here are in good agreement with those reported in [16]. The situation is different with the $3^{\text {rd }}$ harmonic pulse, where both the blue-shift and the spectral broadening are less pronounced, in accordance with the $\Delta \lambda \sim \lambda^{3}$ dependence of the blue-shift as shown in [13].

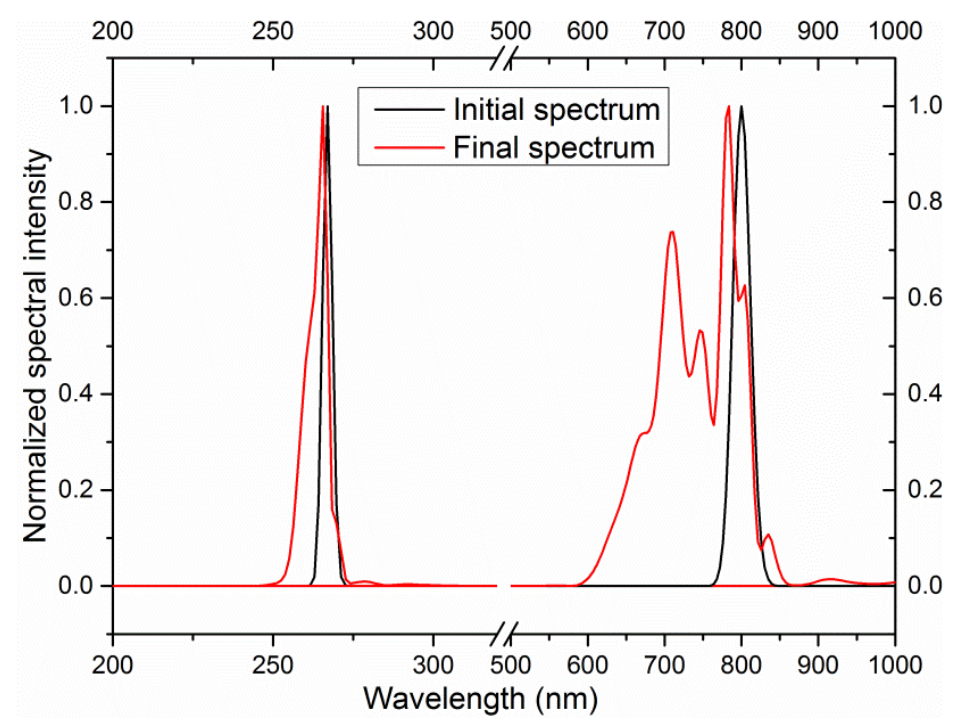

Fig. 5. (color online)

\section{Conclusions}

We have presented a numerical model developed for the description of a single-shot laser pulse propagating through a gas medium which consists of multiply ionized atomic species. The propagation of a laser pulse approaching the relativistic intensity regime induces strong effects in the characteristics of the laser pulse itself, which were explored both in time/frequency and in space domain. In this study we showed detailed results for two characteristic cases, the $800 \mathrm{~nm}$ pulse as well as its third harmonic $267 \mathrm{~nm}$ pulse, both being multi-cycle pulses and operating in single-shot mode.

We performed detailed calculations using a 3D model for pulse propagation and a system of rate equations for the calculation of the electron density which influences the effective refractive index of the medium. The results show that the modifications of the driving field both in temporal/spectral and spatial domain are due to the rapidly changing refractive index in time and space dominated by the corresponding variation of electron plasma. We point out that pulse characteristics achieved during propagation are important in modeling various phenomena of practical use, like harmonic generation and particle acceleration. 
Acknowledgements

VT, KK and DU gratefully acknowledge financial support from Romanian Financing Authority CNDIUEFISCDI projects RO-CERN ELI-NP 03-ELI/2016 (ProPW) and PN-II- PN-II-PT-PCCA-2011-3.1-0886 No. 1/2012 (UFO-UV). VT and KK also acknowledge support from PN-II-RU-TE-2014-4-0425 (185/2015). The project was supported by the Hungarian National Research, Development and Innovation Office (OTKA NN107235). ELI-ALPS is supported by the European Union and co-financed by the European Regional Development Fund (GOP-1.1.1-12/B-2012-000, GINOP-2.3.6-15-2015-00001). 


\section{References}

[1] E. Esarey, C.B. Schroeder, W.P. Leemans, Rev. Mod. Phys. 81, 1229 (2009) DOI:

10.1103/RevModPhys.81.1229

[2] M. Chen, J. Luo, FY. Li, F. Liu, ZM. Sheng, J. Zhang, Light-Science \& Applications 5, e16015 (2016) DOI: $10.1038 / \mathrm{lsa} .2016 .15$

[3] F. Krausz, M. Ivanov, Rev. Mod. Phys. 81, 163 (2009). DOI: 10.1103/RevModPhys.81.163

[4] E.E. Serebryannikov, E. Goulielmakis, A.M. Zheltikov, New J. Phys. 10, 093001 (2008) DOI:

$10.1088 / 1367-2630 / 10 / 9 / 093001$

[5] T. Hosokai, K. Kinoshita, A. Zhidkov, K. Nakamura, T. Watanabe, T. Ueda, H. Kotaki, M. Kando, K. Nakajima, M. Uesaka, Phys. Rev. E 67, 036407 (2003). DOI: 10.1103/PhysRevE.67.036407

[6] H.T. Kim, K.H. Pae, H.J. Cha, I.J. Kim, T.J. Yu, J.H. Sung, S.K. Lee, T.M. Jeong, J. Lee, Phys. Rev. Lett. 111, 165002 (2013) DOI: 10.1103/PhysRevLett.111.165002

[7] B. Beaurepaire, A. Lifschitz and J. Faure, New J. Phys. 16, 023023 (2014) DOI: 10.1088/1367$2630 / 16 / 2 / 023023$

[8] C.M. Heyl, H. Coudert-Alteirac, M. Miranda, M. Louisy, K. Kovacs, V. Tosa, E. Balogh, K. Varjú, A. L'Huillier, A. Couairon, C.L. Arnold, Optica, 3, 75 (2016) DOI: 10.1364/OPTICA.3.000075

[9] P. Arpin, T. Popmintchev, N. Wagner, A.L. Lytle, O. Cohen, M.M. Murnane, and H.C. Kapteyn, Phys. Rev. Lett. 103, 143901 (2009) DOI: 10.1103/PhysRevLett.103.143901

[10] D. Popmintchev, C. Hernández-García, F. Dollar, C. Mancuso, J. A. Pérez-Hernández, M-C. Chen, A. Hankla, X. Gao, B. Shim, A. Gaeta, M. Tarazkar, D. A. Romanov, R. J. Levis, J. A. Gaffney, M. Foord, S. B. Libby, A. J. Becker, A. Becker, L. Plaja, M. M. Murnane, H. C. Kapteyn, T. Popmintchev, Science 350, 1225 (2015) DOI: 10.1126/science.aac9755

[11] V. Tosa, K. T. Kim, and C. H. Nam, Phys. Rev. A 79, 043828 (2009) DOI:

10.1103/PhysRevA.79.043828

[12] P.G.D. Martínez, A.C La Fontaine, C Köhler and L Bergé, J. Phys. B: At. Mol.Opt. Phys. 48, 094010 (2015) DOI: 10.1088/0953-4075/48/9/094010

[13] S.C Rae and K. Burnett, Phys. Rev. A 46, 1084 (1992) DOI: 10.1103/PhysRevA.46.1084 
[14] M.V. Ammosov, N.B. Delone, and V.P. Krainov, Zh. Eksp. Teor. Fiz. 91, 2008 (1986) [Sov. Phys. JETP 64, 1191 (1987)]

[15] P. Sprangle, E. Esarey, and B. Hafizi, Phys. Rev. Lett. 79, 1046 (1997) DOI:

10.1103/PhysRevLett.79.1046

[16] S.P. Nikitin, Y.L. Li, T.M. Antonsen, H.M. Milchberg, Opt. Comm. 157, 139-144 (1998) DOI:

$10.1016 / \mathrm{S} 0030-4018(98) 00473-8$

[17] S.P. Le Blanc and R. Sauerbrey, J. Opt. Soc. Am. B 13, 72-88 (1996) 


\section{List of figure captions}

Fig. 1 (color online) Population fraction of ions with increasing charge showing how they appear, saturate and disappear (through further ionization) in time during the rising part of the $800 \mathrm{~nm}$ laser pulse. The envelope of the laser pulse is also shown for identifying the "lifetime" of ions of given charge.

Fig. 2 (color online) Ionization degree as a function of time at the entrance of the Ar medium (black / top) and after $2 \mathrm{~mm}$ propagation (red / bottom). Left: $800 \mathrm{~nm}$ pulse, right: $267 \mathrm{~nm}$ pulse. The full electric fields of the initial and final laser pulses are also shown. Individual ionization steps happen every half optical cycle. The bigger steps are also visible when ions of $\mathrm{Z}$ charge are depleted and ions with $\mathrm{Z}+1$ charge start to dominate.

Fig. 3 (color online) 3D map of the spatial evolution of the peak intensities for the two laser pulses. The inset graph shows the laser pulse intensity envelope at the three positions indicated by dots of respective colors from the 3D map.

Fig. 4 (color online) Spatial map of the ionization degree right at the pulse end, for both cases. Left: ionization degree produced by the $800 \mathrm{~nm}$ pulse; right: the same for the $267 \mathrm{~nm}$ pulse.

Fig. 5 (color online) Initial and final spectra of the two laser fields. All spectra are normalized. 